\title{
Supplementary document for \\ Colossal crystals in P3HT:PCBM blends for enhanced organic magnetism
}

\author{
Scott Newacheck, Nha Uyen Huynh, and George Youssef \\ San Diego State University, San Diego, U.S.A
}

\begin{abstract}
A series of photographs were taken to illustrate the process of fabricating the thick P3HT:PCBM films, with and without acetonitrile. Following the solution preparation process outlined in the main document, a relatively large volume of the solution with respect to the substrate dimensions was drop-casted onto $\mathrm{Si}$ and ITO substrates. Figure S1 shows an exemplary photograph of the P3HT:PCBM solution drop-casted onto an ITO substrate. The solutions were then allowed to cure in a controlled environment, where the cured samples are shown in Figure S2. The samples fabricated without acetonitrile (Figure S2a) were covered with various surface features that can be observed upon close inspection. Conversely, the samples fabricated with acetonitrile (Figure S2b) were relatively smooth with only a few observable surface features present on the silicon substrate, but none of which possessed a well-defined rhombohedral shape.
\end{abstract}

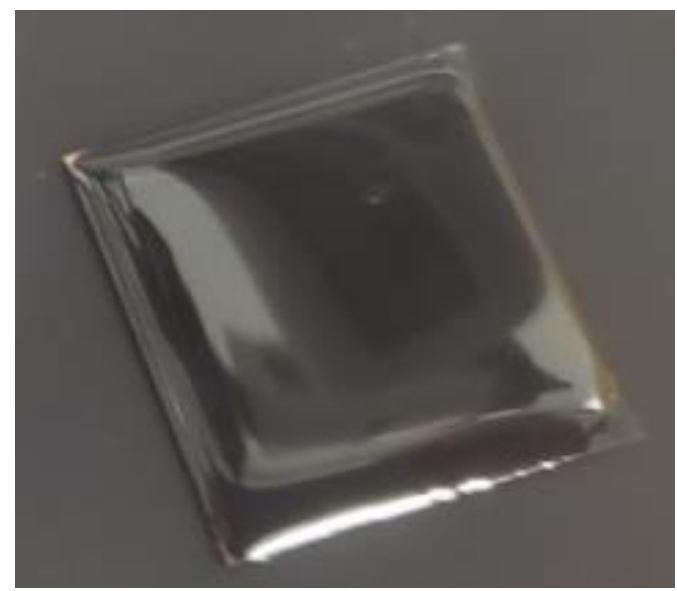

Figure S1: Photo of the P3HT:PCBM solution deposited on cleaned ITO glass substrate. 

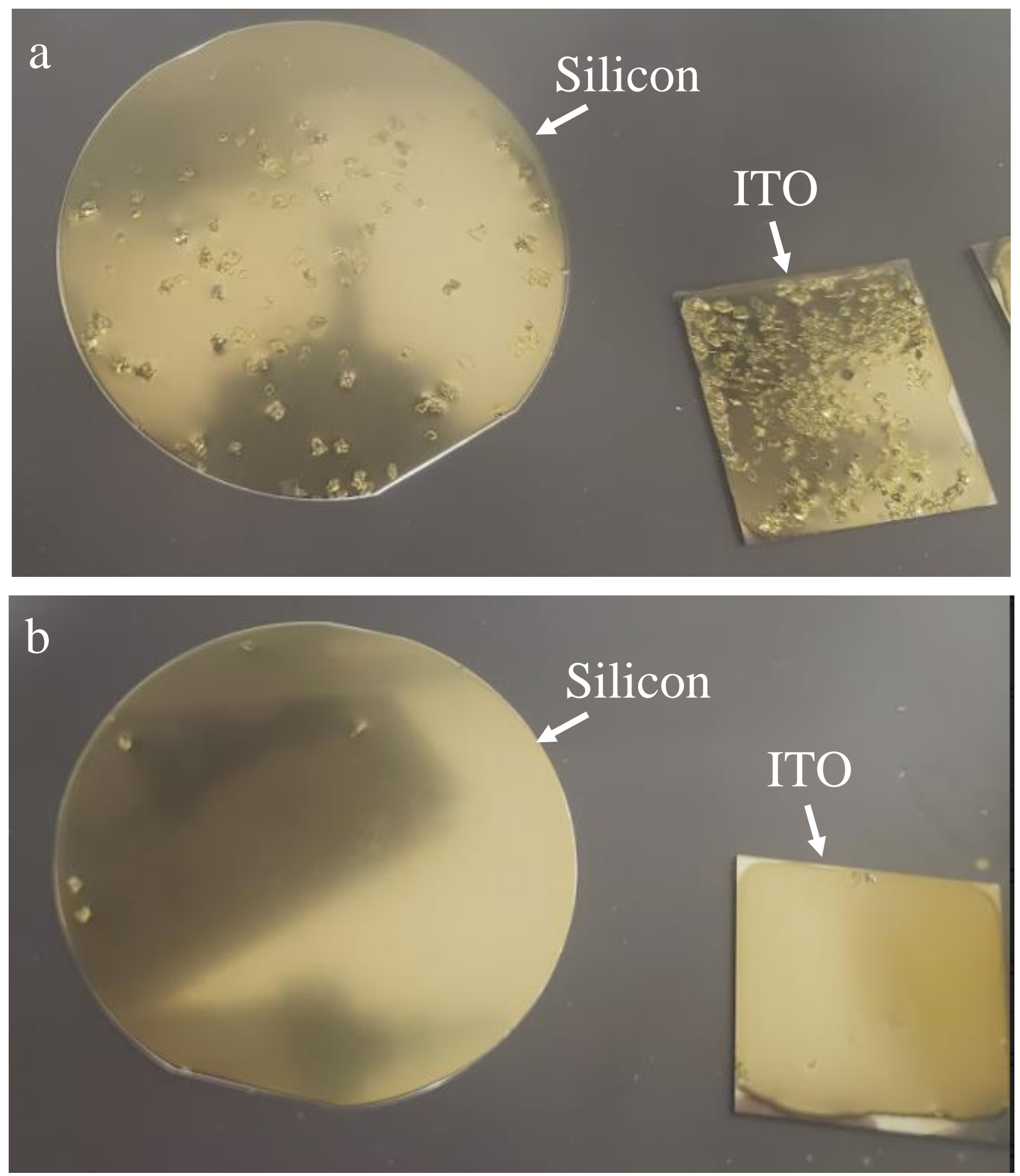

Figure S2: photos of (a) without acetonitrile and (b) with acetonitrile drop-casted P3HT:PCBM cured on silicon and ITO substrates. 
To acquire the scanning electron microscopy images (e.g., Figure 1 in the main document), a 6 nm layer of platinum was sputtered on a set of samples fabricated with and without ACN on the ITO substrate, illuminating the solvent dependent morphological behaviors using a FEI Quanta 450 scanning electron microscope (SEM). Figure S3 shows an SEM image of multiple millimeter-scale crystals on the nonacetonitrile sample, demonstrating replicability.

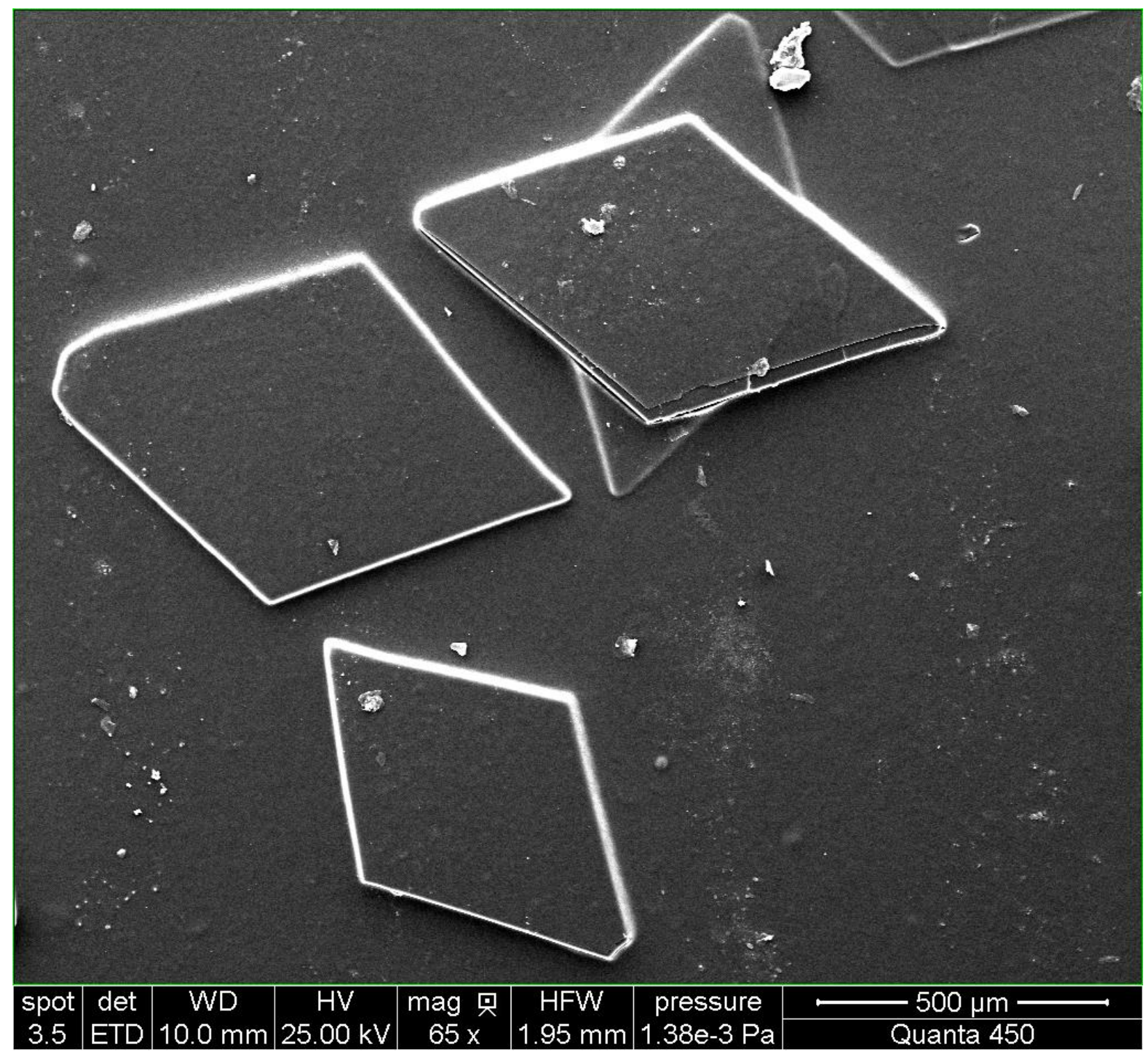

Figure S3: scanning electron microscope micrograph of multiple millimeter-scale PCBM crystals in the non-acetonitrile sample. 
Additional MFM scans of the non-acetonitrile sample are shown in Figure S4. The magnetic domain characteristic of the off-crystal structure was similar to the sample with fabricated with ACN (shown in main document). However, no observable magnetic response was more common for the off-crystal surface.

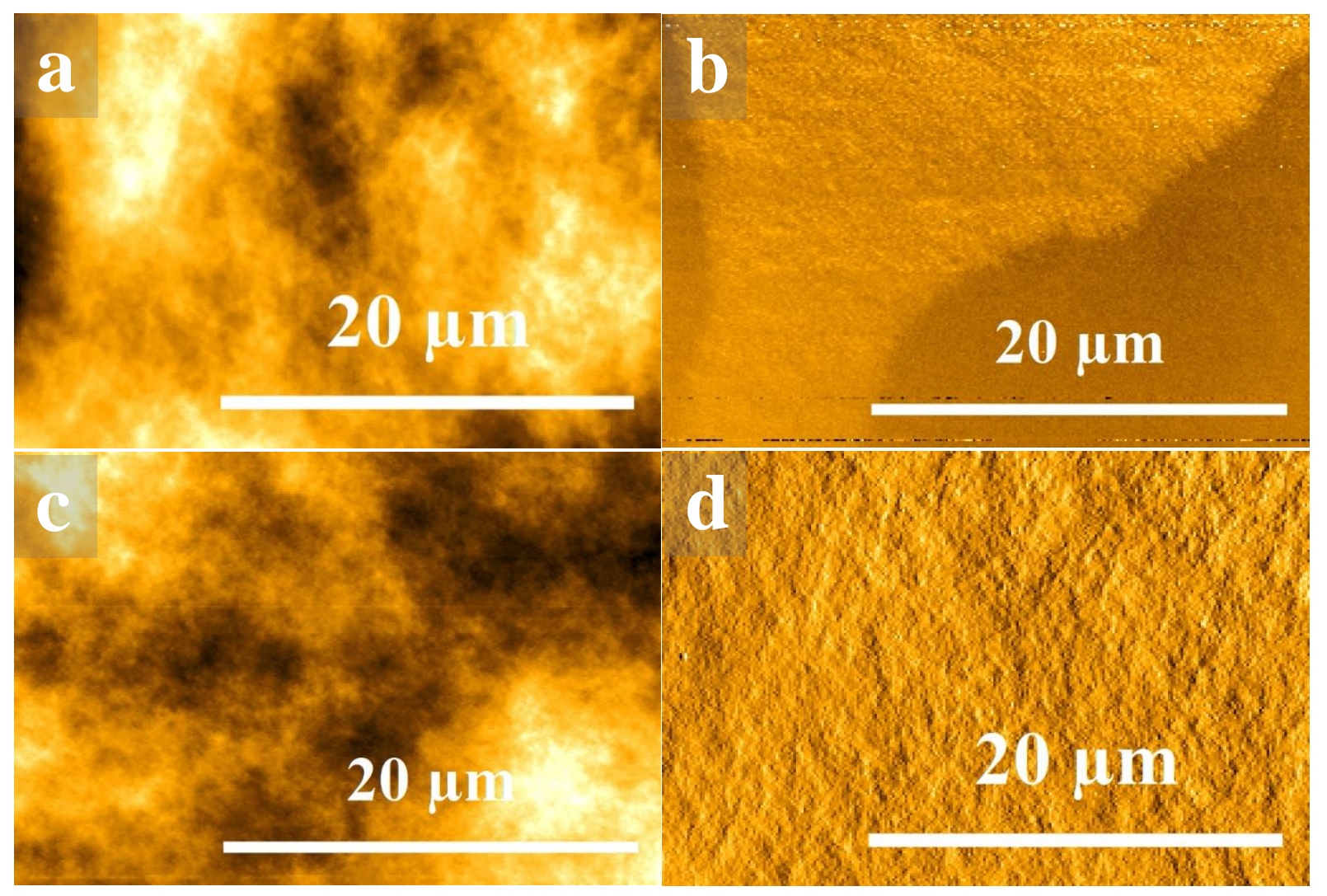

Figure S4: MFM results (left column is topography and right column is magnetic phase) for samples fabricated without ACN, (a \& b) on and (c \& d) off the PCBM crystal. 